\title{
Indirect effect of the triaxiality in the Hamiltonian theory for the rigid Earth nutations
}

\author{
A. Escapa ${ }^{1}$, J. Getino $^{2}$, and J. M. Ferrándiz ${ }^{1}$ \\ 1 Dpto. de Matemática Aplicada, Escuela Politécnica Superior, Universidad de Alicante, 03080 Alicante, Spain \\ 2 Grupo de Mecánica Celeste, Facultad de Ciencias, Universidad de Valladolid, 47005 Valladolid, Spain \\ Received 20 September 2001 / Accepted 30 April 2002
}

\begin{abstract}
In this investigation we determine a new contribution to the nutation series due to an indirect effect of the triaxiality of the rigid Earth. We undertake this work in the context of the Hamiltonian theory for the rigid Earth, developed by Kinoshita (1977) and recently updated by Souchay et al. (1999). To do this, we express the potential energy in terms of the action-angle variables for the triaxial Earth. Then, we work out analytical formulae for the new contributions, showing that their numerical values are within the truncation level adopted by REN-2000 (Souchay et al. 1999), which was fixed to $0.1 \mu$ as.
\end{abstract}

Key words. celestial mechanics

\section{Introduction}

In the last years, a group leaded by Dr. Souchay has carried out an extensive reconstruction of the Hamiltonian theory of the nutation for the rigid Earth (Souchay \& Kinoshita 1996, 1997; Folgueira et al. 1998a, 1998b; Souchay et al. 1999). The purpose of these studies was to update the pioneering investigations of Kinoshita (1977) and Kinoshita \& Souchay (1990), in order to achieve a precision at the level of $0.1 \mu$ as (microarcsecond) for each individual term of the nutation series of the Earth. Hence, it has been necessary to perform successive enhancements in the basic rigid Earth theory by recalculating more precisely known contributions, or by including new effects previously disregarded, like the study of coupling mechanisms, influence of the planets, etc. (see Souchay et al. 1999 or Souchay 2000, for a complete description). The results of these works are collected in Souchay et al. (1999), where the tables for the resulting rigid Earth nutation (REN-2000) seemed to be definitive. As it is pointed out by Souchay et al. (1999), the tables REN-2000 "catch all the coefficients up to $0.1 \mu \mathrm{as"}$.

One of the mentioned enhancements consists of incorporating the effects of the triaxiality of the Earth. As it is well-known, the Earth is not a perfect symmetrical body; this fact gives rise to the appearance of the non-zonal harmonics of the geopotential expansion, which affects the Earth nutations causing the so-called short period nutations, mainly diurnal and subdiurnal. The in-

Send offprint requests to: A. Escapa,

e-mail: Alberto.Escapa@ua.es fluence of these terms was studied by Kinoshita (1977), Kinoshita \& Souchay (1990), Souchay \& Kinoshita (1997) and Souchay et al. (1999) for the $C_{22}$ and $S_{22}$ coefficients and by Folgueira et al. (1998a, 1998b) for the nonzonal coefficients of third and fourth degree. Indeed, as it is remarked in Souchay (2000), the short-period nutations terms are one of the most important new contributions allowing the REN-2000 tables to reach the submicroarsecond level.

Those works include the effect of the triaxiality in a direct way, that is to say, they compute the nutations terms arising from the potential terms caused by the triaxiality (non-zonal harmonics). However, the triaxiality also modifies the expression of the kinetic energy that is fundamental in the application of perturbation methods. Because of this fact, the action-angle variables for the torque-free motion of a triaxial Earth do not coincide with the Andoyer variables (Kinoshita 1972), which are actionangle variables only for the free motion of a symmetrical rigid body. Since Andoyer variables are not action-angle variables, to apply the Hori perturbation method (Hori 1966) it is convenient to expand the disturbing function in terms of the action-angle variables and not in terms of the Andoyer variables, to avoid the occurrence of elliptical functions when computing the generating function.

This step was published by Kinoshita (1977) himself, but he decided rightly not to take it into account since the truncation level of his work was 0.1 mas (miliarcsecond). The transformation is achieved with the help of a canonical transformation which relates Andoyer variables with the action-angle variables (Kinoshita 1972, 1977). Thus, the triaxiality affects all the terms of the 
potential, the zonal and the non-zonal ones, changing the functional dependence of the disturbing function to some extent. Following the classical nomenclature in Celestial Mechanics we name this effect the "indirect effect" of the triaxiality. So, the triaxiality of the Earth contributes to the nutations in two ways. First, through a "direct effect" due to the existence of the non-zonal harmonics. Second, through an "indirect effect" due to the fact that the solution of the unperturbed problem (free motion) is different from the solution of the symmetrical case. As far as we know, the previous studies in the Hamiltonian theory for the rotation of the rigid Earth have not treated this second kind of influence, which could provide contributions to the nutation series within the present level of accuracy. The first effect has already been calculated by Kinoshita \& Souchay (1990), Souchay \& Kinoshita (1997), Souchay et al. (1999) and Folgueira et al. (1998a, 1998b) and it will not be treated in this work. On the other hand, Getino et al. (2001) computed the "direct effect" of the $C_{22}$ and $S_{22}$ harmonics for a two-layer, non-rigid Earth model.

The present investigation deals with the study of the indirect effect of the triaxiality, following the Hamiltonian method developed by Kinoshita (1977). In particular, we evaluate the contribution to the nutations of this effect when considering the zonal harmonic of the second degree, which is the larger coefficient in the geopotential expansion of the Earth. Although in REN-2000 Souchay et al. (1999) performed a comprehensive reconstruction of the Hamiltonian theory of the rigid Earth, to get the $0.1 \mu$ as truncation level, this effect has not been discussed. However, the numerical contribution of this effect could reach a few tenths of microarcseconds and, therefore, it cannot be neglected within the truncation level sought nowadays.

In Sect. 2 we summarize the free-motion of a rigid body in terms of the action-angle variables associated with the Andoyer variables. In Sect. 3 we express the potential energy with the help of these variables. Once the potential energy has been transformed, in Sect. 4 we apply Hori's perturbation method and derive the generating function. In addition, we take advantage of the canonical invariance of this perturbation method and re-express the generating function as a function of the Andoyer variables that are more convenient for the calculations. In Sect. 5 we compute the analytical formulae for the nutation of the Andoyer plane, i.e. the plane perpendicular to the angular momentum axis of the Earth, the equatorial plane, i.e. the plane perpendicular to the figure axis of the Earth, and the plane perpendicular to the rotational axis. Finally, by using the same numerical parameters as in REN-2000 (Souchay et al. 1999) we compute the numerical expressions for the nutation series. We find that sixteen terms, eight for the nutation of the equatorial plane and eight for the nutation of the rotational axis, are larger than $0.05 \mu$ as, with six terms larger than $0.1 \mu$ as.

\section{Torque-free motion}

Next, we give a brief description of the solution of the torque-free motion in the short-axis rotation mode of a rigid body. We follow the same notation as in Kinoshita (1977). A more comprehensive study can be found in Kinoshita (1972, 1977).

The dynamical behavior of this system is described by means of the Andoyer canonical variables: $G, L, H, g, l$, $h$ and the auxiliary angles $J(L=G \cos J)$ and $I(H=$ $G \cos I)$. A dynamical and geometrical interpretation of this set is detailed in Kinoshita (1977). The mechanical properties of the rigid body, the Earth in our case, are characterized by its moments of inertia $A<B<C$. At this point, it is useful to introduce the non dimensional parameters (Kinoshita 1977)

$H_{\mathrm{d}}=\frac{2 C-A-B}{2 C}, e=\frac{1 /(2 B)-1 /(2 A)}{1 / C-1 /(2 A)-1 /(2 B)}$.

$H_{\mathrm{d}}$ is the dynamical ellipticity of the body and $e$ is a measure of the triaxiality, this parameter characterizing the departure from a symmetrical body $(A=B)$. For the Earth $e \sim 10^{-3}$ so, in this work we suppose that $e$ is a small parameter, and we only retain terms up to the first order in $e$.

The Hamiltonian for torque-free motion is given (Kinoshita 1977) by

$F_{0}=\frac{1}{2}\left(\frac{\sin ^{2} l}{A}+\frac{\cos ^{2} l}{B}\right)\left(G^{2}-L^{2}\right)+\frac{1}{2} \frac{L^{2}}{C}$.

As it can be seen, the Hamiltonian only depends on the coordinate $l$ and the system can be solved by quadratures, leading to elliptic functions. We recall that the action-angle variables of a problem are characterized by the fact that the canonical momenta (action variables) are constants of motion while the canonical variables (angle variables) evolve linearly with time (Goldstein 1980). Therefore, Andoyer variables are not angle-action ones for the triaxial rigid body due to the occurrence of the coordinate $l$ in Eq. (2), unlike the axis-symmetrical case $(A=B)$ in which $l$ disappears.

The action-angle set for the torque-free motion of a triaxial rigid body is developed by Kinoshita (1972), where a construction of these variables based on Andoyer variables is given. Though the canonical transformation relating both canonical sets (action-angle variables and Andoyer variables) involves the use of elliptical functions, it is possible to obtain more suitable expressions by expanding the canonical transformation in terms of $e$, which as we have pointed out is a small parameter. Thus, from Kinoshita (1977) we have

$$
\begin{aligned}
& l=\tilde{l}-\frac{1}{4} e\left(1+\sec ^{2} \tilde{J}\right) \sin 2 \tilde{l}, g=\tilde{g}+\frac{1}{2} e \sec \tilde{J} \sin 2 \tilde{l}, \\
& J=\tilde{J}+\frac{1}{2} e \tan \tilde{J} \cos 2 \tilde{l}, G=\tilde{G}, H=\tilde{H}, h=\tilde{h}
\end{aligned}
$$


where $\tilde{G}, \tilde{L}, \tilde{g}$ and $\tilde{l}$ are the action-angle variables corresponding to $G, L, g$ and $l$ and $\cos \tilde{J}=\tilde{L} / \tilde{G}$. The mean motions $n_{\tilde{l}}$ and $n_{\tilde{g}}$ of $\tilde{l}$ and $\tilde{g}$ (Kinoshita 1972) are

$n_{\tilde{g}}=\frac{1}{2}\left(\frac{1}{A}+\frac{1}{B}\right) \tilde{G}, n_{\tilde{l}}=\left(\frac{1}{C}-\frac{1}{2 A}-\frac{1}{2 B}\right) \tilde{L}$,

whose expressions coincide, in our level of approximation, with the mean motions of $l$ and $g$ (Kinoshita 1977).

\section{Disturbing function in terms of action-angle variables}

As is pointed in Kinoshita (1977), in order to take into account the triaxiality in a complete way, we have to express the potential energy, or disturbing function, in terms of the action-angle variables. Because the triaxiality is small and the related geopotential coefficients consequently are also small, we only consider this transformation in the largest term of the geopotential, that is to say, in the zonal harmonic of the second degree $\left(J_{2}\right.$ term). From Kinoshita (1977) we have

$$
\begin{aligned}
V= & \frac{k^{\prime} a^{3}}{r^{3}} P_{2}(\sin \delta)=\frac{k^{\prime}}{2}\left(3 \cos ^{2} J-1\right) \sum_{\nu} B_{\nu} \cos \Theta_{\nu} \\
& -\frac{k^{\prime}}{2} \sin 2 J \sum_{\nu} \sum_{\varepsilon= \pm 1} C_{\nu}(\varepsilon) \cos \left(g-\varepsilon \Theta_{\nu}\right) \\
& +\frac{k^{\prime}}{4} \sin ^{2} J \sum_{\nu} \sum_{\varepsilon= \pm 1} D_{\nu}(\varepsilon) \cos \left(2 g-\varepsilon \Theta_{\nu}\right),
\end{aligned}
$$

where $k^{\prime}=3 \kappa^{2} M^{\prime} a^{-3}(2 C-A-B) / 2 . k^{\prime}$ is a numerical constant that depends on the perturbing body, Moon or Sun. Let us remember that $V$ depends on $I$ through $B_{\nu}$, $C_{\nu}(\varepsilon)$ and $D_{\nu}(\varepsilon)$ and on $h$ through $\Theta_{\nu}$ (Kinoshita 1977). Substituting the relationships (3) in (5) we get

$$
\begin{aligned}
\tilde{V}= & \frac{k^{\prime}}{2}\left(3 \cos ^{2} \tilde{J}-1\right) \sum_{\nu} B_{\nu} \cos \Theta_{\nu} \\
& -\frac{k^{\prime}}{2} \sin 2 \tilde{J} \sum_{\nu} \sum_{\varepsilon= \pm 1} C_{\nu}(\varepsilon) \cos \left(\tilde{g}-\varepsilon \Theta_{\nu}\right) \\
& +\frac{k^{\prime}}{4} \sin ^{2} \tilde{J} \sum_{\nu} \sum_{\varepsilon= \pm 1} D_{\nu}(\varepsilon) \cos \left(2 \tilde{g}-\varepsilon \Theta_{\nu}\right) \\
& -\frac{3 k^{\prime} e}{4} \sin ^{2} \tilde{J} \sum_{\nu} \sum_{\varepsilon= \pm 1} B_{\nu} \cos \left(2 \tilde{l}-\varepsilon \Theta_{\nu}\right) \\
& -\frac{k^{\prime} e}{4} \sin \tilde{J} \sum_{\nu} \sum_{\rho, \varepsilon= \pm 1} C_{\nu}(\varepsilon)\left(\rho+\frac{\cos 2 \tilde{J}}{\cos \tilde{J}}\right) \\
& \times \cos \left(\tilde{g}+2 \rho \tilde{l}-\varepsilon \Theta_{\nu}\right) \\
& +\frac{k^{\prime} e}{8} \sin \tilde{J} \tan \tilde{J} \sum_{\nu} \sum_{\rho, \varepsilon= \pm 1} D_{\nu}(\varepsilon)(\rho+\cos \tilde{J}) \\
& \times \cos \left(2 \tilde{g}+2 \rho \tilde{l}-\varepsilon \Theta_{\nu}\right) .
\end{aligned}
$$

The transformed function has two parts. The first one has the same functional dependence as Eq. (5), but the
Andoyer variables have been literally replaced by the action-angle variables. The second one is a new term which arises as a direct consequence of incorporating the triaxiality. Note that if the rigid body were symmetrical then $e=0$ and the expression of the disturbing function in terms of the action-angle variables would coincide with the expression of the disturbing function in terms of the Andoyer variables. This is a consequence of the fact that when $e=0$ Andoyer variables are the action-angle variables for the free motion of a symmetrical rigid body.

\section{Generating function}

In order to carry out the analytical integration of the problem, Kinoshita (1977) applies a perturbation technique based on the Lie series (Hori 1966). With this technique, it is necessary to compute the generating function of a canonical transformation, so that the problem will be easier to solve in terms of the new canonical variables resulting from this transformation. The generating function is obtained by means of integrating the periodic part of the potential energy along the solutions of the unperturbed problem. From Eqs. (3) and (6) it follows that the secular part of $V$ is not altered by the transformation into action-angle variables, that is to say:

$$
\begin{aligned}
\tilde{V}_{\mathrm{sec}} & =\frac{k^{\prime}}{2}\left(3 \cos ^{2} \tilde{J}-1\right) B_{0} \cos \Theta_{0} \\
& =\frac{k^{\prime}}{2}\left(3 \cos ^{2} J-1\right) B_{0} \cos \Theta_{0}=V_{\mathrm{sec}}
\end{aligned}
$$

therefore, the generating function will be given by

$\tilde{W}=\int\left(\tilde{V}-\tilde{V}_{\mathrm{sec}}\right) \mathrm{d} t$.

The calculus of this integral is straightforward, providing

$$
\begin{aligned}
\tilde{W}= & \frac{k^{\prime}}{2}\left(3 \cos ^{2} \tilde{J}-1\right) \sum_{\nu \neq 0} \frac{B_{\nu}}{N_{\nu}} \sin \Theta_{\nu} \\
& -\frac{k^{\prime}}{2} \sin 2 \tilde{J} \sum_{\nu} \sum_{\varepsilon= \pm 1} \frac{C_{\nu}(\varepsilon)}{n_{\tilde{g}}-\varepsilon N_{\nu}} \sin \left(\tilde{g}-\varepsilon \Theta_{\nu}\right) \\
& +\frac{k^{\prime}}{4} \sin ^{2} \tilde{J} \sum_{\nu} \sum_{\varepsilon= \pm 1} \frac{D_{\nu}(\varepsilon)}{2 n_{\tilde{g}}-\varepsilon N_{\nu}} \sin \left(2 \tilde{g}-\varepsilon \Theta_{\nu}\right) \\
& -\frac{3 k^{\prime}}{4} e \sin ^{2} \tilde{J} \sum_{\nu} \sum_{\varepsilon= \pm 1} \frac{B_{\nu}}{2 n_{\tilde{l}}-\varepsilon N_{\nu}} \sin \left(2 \tilde{l}-\varepsilon \Theta_{\nu}\right) \\
& -\frac{k^{\prime}}{4} e \sin \tilde{J} \sum_{\nu} \sum_{\rho, \varepsilon= \pm 1} C_{\nu}(\varepsilon)\left(\rho+\frac{\cos 2 \tilde{J}}{\cos \tilde{J})}\right. \\
& \times \frac{1}{n_{\tilde{g}}+2 \rho n_{\tilde{l}}-\varepsilon N_{\nu}} \sin \left(\tilde{g}+2 \rho \tilde{l}-\varepsilon \Theta_{\nu}\right) \\
& +\frac{k^{\prime}}{8} e \sin \tilde{J} \tan \tilde{J} \sum_{\nu} \sum_{\rho, \varepsilon= \pm 1} D_{\nu}(\varepsilon)(\rho+\cos \tilde{J}) \\
& \times \frac{1}{2 n_{\tilde{g}}+2 \rho n_{\tilde{l}}-\varepsilon N_{\nu}} \sin \left(2 \tilde{g}+2 \rho \tilde{l}-\varepsilon \Theta_{\nu}\right),
\end{aligned}
$$

with $N_{\nu}=\dot{\Theta}_{\nu}$. In this integration, we have neglected the contribution coming from the secular part of the obliquity, 
$I$, and of the coefficients $B_{\nu}, C_{\nu}(\varepsilon)$ and $D_{\nu}(\varepsilon)$, because their effects are negligible as far as the nutation due to the indirect effect of the triaxiality is concerned. In addition, to simplify the notation we have noted with the same symbol the canonically transformed variables. It is must be understood that the variables in the right hand side of Eq. (9) are the transformed variables.

We compute the forced nutations of the Earth with the aid of $\tilde{W}$, by expressing the longitude and obliquity of the Andoyer plane, the equatorial plane, and the plane perpendicular to the rotational axis in terms of the actionangle variables. Anyway, we can take advantage of the canonical invariance of the Hori's method (Hori 1966) by re-expressing the generating function, Eq. (9), in terms of the Andoyer variables. Following this procedure, the contributions of the indirect effect of the triaxiality to the nutations, computed in this work, may be added directly to REN-2000 tables (Souchay et al. 1999).

To do this, we have to find the inverse relationships of Eqs. (3). These relations turn out to be

$\tilde{l}=l+\frac{1}{4} e\left(1+\sec ^{2} J\right) \sin 2 l, \tilde{g}=g-\frac{1}{2} e \sec J \sin 2 l$,

$\tilde{J}=J-\frac{1}{2} e \tan J \cos 2 l, \tilde{G}=G$.

Substituting these equations in (9) we obtain

$W=W_{K}+e W_{\mathrm{e}}$,

where

$$
\begin{aligned}
W_{K}= & \frac{k^{\prime}}{2}\left(3 \cos ^{2} J-1\right) \sum_{\nu} \frac{B_{\nu}}{N_{\nu}} \sin \Theta_{\nu} \\
& -\frac{k^{\prime}}{2} \sin 2 J \sum_{\nu} \sum_{\varepsilon= \pm 1} \frac{C_{\nu}(\varepsilon)}{n_{g}-\varepsilon N_{\nu}} \sin \left(g-\varepsilon \Theta_{\nu}\right) \\
& +\frac{k^{\prime}}{4} \sin ^{2} J \sum_{\nu} \sum_{\varepsilon= \pm 1} \frac{D_{\nu}(\varepsilon)}{2 n_{g}-\varepsilon N_{\nu}} \sin \left(2 g-\varepsilon \Theta_{\nu}\right) .
\end{aligned}
$$

This is the same expression that Kinoshita (1977) got when computing the generating function over the solution of the symmetric unperturbed problem. On the other hand, we have

$$
\begin{aligned}
W_{\mathrm{e}}= & -\frac{3 k^{\prime}}{2} \sin ^{2} J \sum_{\nu \neq 0} \sum_{\varepsilon= \pm 1} \frac{\varepsilon B_{\nu} n_{l}}{N_{\nu}\left(2 n_{l}-\varepsilon N_{\nu}\right)} \sin \left(2 l-\varepsilon \Theta_{\nu}\right) \\
& -\frac{3 k^{\prime}}{4} \sin ^{2} J \frac{B_{0}}{n_{l}} \sin 2 l \\
& -\frac{k^{\prime}}{2} \sin J \sum_{\nu} \sum_{\rho, \varepsilon= \pm 1} C_{\nu}(\varepsilon)\left(\rho+\frac{\cos 2 J}{\cos J}\right) \\
& \times \frac{\rho n_{l} \sin \left(g+2 \rho l-\varepsilon \Theta_{\nu}\right)}{\left(n_{g}-\varepsilon N_{\nu}\right)\left(n_{g}+2 \rho n_{l}-\varepsilon N_{\nu}\right)} \\
& -\frac{k^{\prime}}{4} e \sin J \tan J \sum_{\nu} \sum_{\rho, \varepsilon= \pm 1} D_{\nu}(\varepsilon)(\rho+\cos J) \\
& \times \frac{\rho n_{l} \sin \left(2 g+2 \rho l-\varepsilon \Theta_{\nu}\right)}{\left(2 n_{g}-\varepsilon N_{\nu}\right)\left(2 n_{g}+2 \rho n_{l}-\varepsilon N_{\nu}\right)} .
\end{aligned}
$$

In both expressions we have taken into account that $n_{\tilde{g}}=$ $n_{g}$ and $n_{\tilde{l}}=n_{l}$.

\section{Nutations of the fundamental planes}

Next, we compute the nutations for the three fundamental planes in the theory of the rotation of the Earth. These are the plane normal to the angular momentum (Andoyer plane), the plane normal to the figure axis (equatorial plane) and the plane normal to the instantaneous rotational axis. As usual (see, for instance, Kinoshita 1977 or Souchay et al. 1999), the nutations are computed to order 0 in $J$, since $J \sim 10^{-6}$ rd (Kinoshita 1977).

Let us remember that the periodic variations of any function, due to the perturbation, are given to the first order by Hori (1966)

$\Delta f=\{f, S\}$

In this expression $f$ is a function of the canonical variables, $S$ is the generating function of the first order and $\{$,$\} is$ the symbol representing the Poisson bracket

$$
\begin{aligned}
\{f, S\}= & \frac{\partial f}{\partial g} \frac{\partial S}{\partial G}-\frac{\partial f}{\partial G} \frac{\partial S}{\partial g}+\frac{\partial f}{\partial l} \frac{\partial S}{\partial L}-\frac{\partial f}{\partial L} \frac{\partial S}{\partial l} \\
& +\frac{\partial f}{\partial h} \frac{\partial S}{\partial H}-\frac{\partial f}{\partial H} \frac{\partial S}{\partial h} .
\end{aligned}
$$

It is more expedient to compute the derivatives with respect to the momenta $(G, L, H)$ through the auxiliary angles $I$ and $J$ with the aid of the relationships (Kinoshita 1977):

$$
\begin{aligned}
& \frac{\partial J}{\partial L}=-\frac{1}{G \sin J}, \frac{\partial J}{\partial G}=\frac{\cot J}{G} \\
& \frac{\partial I}{\partial H}=-\frac{1}{G \sin I}, \frac{\partial I}{\partial G}=\frac{\cot I}{G}
\end{aligned}
$$

In this work we are interested in the influence of the triaxiality, therefore, we only focus our attention on the nutations terms that are proportional to $e$. Specifically, the functions $f$ and $S$ will be written as

$f=f_{\mathrm{s}}+e f_{\mathrm{e}}, S=S_{\mathrm{s}}+e S_{\mathrm{e}}$,

and the nutations will be

$\Delta f=\{f, S\}=\left\{f_{\mathrm{s}}, S_{\mathrm{s}}\right\}+e\left(\left\{f_{\mathrm{s}}, S_{\mathrm{e}}\right\}+\left\{f_{\mathrm{e}}, S_{\mathrm{s}}\right\}\right)+O\left(e^{2}\right)$.

The first and leading term on the right hand side of this equation has been treated in REN-2000 (Souchay et al. 1999), Kinoshita \& Souchay (1990) and Kinoshita (1977). The second term is the new contribution due to the indirect effect of the triaxiality. So, we only work out expressions of the form

$\Delta_{e} f=e\left(\left\{f_{\mathrm{s}}, S_{\mathrm{e}}\right\}+\left\{f_{\mathrm{e}}, S_{\mathrm{s}}\right\}\right)$.

\subsection{Nutations of the Andoyer plane}

The node and the inclination of this plane are given by the angles $h$ and $I$, respectively. So, we have

$\Delta h=\{h, W\}=\left\{h, W_{\mathrm{K}}\right\}+e\left\{h, W_{\mathrm{e}}\right\}$,

$\Delta I=\{I, W\}=\left\{I, W_{\mathrm{K}}\right\}+e\left\{I, W_{\mathrm{e}}\right\}$, 
taking into account Eqs. (13), (15) and (16) we obtain

$\Delta_{\mathrm{e}} h=O(J)=0, \Delta_{\mathrm{e}} I=O(J)=0$.

Therefore, the nutations in longitude and obliquity of the Andoyer plane, called Poisson terms (Kinoshita 1977), are not affected by the indirect effect of the triaxiality.

\subsection{Nutations of the equatorial plane}

The node and the inclination of this plane are given by the angles $h_{\mathrm{f}}$ and $I_{\mathrm{f}}$, respectively. These angles are written in terms of the Andoyer set as (Kinoshita 1977)

$h_{\mathrm{f}}=h+\frac{J \sin g}{\sin I}+O\left(J^{2}\right), I_{f}=I+J \cos g+O\left(J^{2}\right)$.

The nutations of this plane will be given by

$\Delta h_{\mathrm{f}} \approx\left\{h_{\mathrm{f}}, W\right\}=\Delta h+\left\{\frac{J \sin g}{\sin I}, W_{\mathrm{K}}\right\}+e\left\{\frac{J \sin g}{\sin I}, W_{\mathrm{e}}\right\}$,

$\Delta I_{\mathrm{f}} \approx\left\{I_{\mathrm{f}}, W\right\}=\Delta I+\left\{J \cos g, W_{\mathrm{K}}\right\}+e\left\{J \cos g, W_{\mathrm{e}}\right\}$.

From Eqs. (13), (15), (16), (21) and (23) we have

$\Delta_{\mathrm{e}} h_{\mathrm{f}}=\frac{e k}{\sin I} \sum_{\nu} \sum_{\varepsilon= \pm 1} \frac{n_{l} C_{\nu}(\varepsilon) \sin \left(2 g+2 l-\varepsilon \Theta_{\nu}\right)}{\left(n_{g}-\varepsilon N_{\nu}\right)\left(n_{g}+2 n_{l}-\varepsilon N_{\nu}\right)}$,

$\Delta_{\mathrm{e}} I_{\mathrm{f}}=e k \sum_{\nu} \sum_{\varepsilon= \pm 1} \frac{n_{l} C_{\nu}(\varepsilon) \cos \left(2 g+2 l-\varepsilon \Theta_{\nu}\right)}{\left(n_{g}-\varepsilon N_{\nu}\right)\left(n_{g}+2 n_{l}-\varepsilon N_{\nu}\right)}$,

where $k=k^{\prime} / G$. The differential nutation of the equatorial plane with respect to the nutation of the Andoyer plane is given by the so-called Oppolzer terms of the figure axis $\left(\right.$ Kinoshita 1977), $\Delta_{\mathrm{e}}(J \sin g / \sin I)$ and $\Delta_{\mathrm{e}}(J \cos g)$ in our case. Oppolzer terms in the figure axis provides the entire indirect contribution to the nutation of the figure axis, since as given our order of approximation Poisson terms are $\Delta_{\mathrm{e}} h=\Delta_{\mathrm{e}} I=0$. It is also worthy noting that these terms have a quasi semi-diurnal period, since $n_{g}+n_{l}=\omega_{\mathrm{E}}, \omega_{\mathrm{E}}$ being the mean angular velocity of the rotation of the Earth (Kinoshita 1977).

\subsection{Nutations of the plane perpendicular to the rotational axis}

The node and the inclination of this plane are given by the angles $h_{\mathrm{r}}$ and $I_{\mathrm{r}}$, respectively. These angles are written in terms of the Andoyer set as (Kinoshita 1977)

$$
\begin{aligned}
h_{\mathrm{r}}= & h+\left(1-\frac{C}{2 A}-\frac{C}{2 B}\right) \frac{J \sin g}{\sin I} \\
& -e\left(1-\frac{C}{2 A}-\frac{C}{2 B}\right) \frac{J \sin (2 l+g)}{\sin I}+O\left(J^{2}\right), \\
I_{\mathrm{r}}= & I+\left(1-\frac{C}{2 A}-\frac{C}{2 B}\right) J \cos g \\
& -e\left(1-\frac{C}{2 A}-\frac{C}{2 B}\right) J \cos (2 l+g)+O\left(J^{2}\right) .
\end{aligned}
$$

These relationships depend on the structure of the body through the parameter $e$. The nutations of this plane are given by

$$
\begin{aligned}
\Delta h_{\mathrm{r}} \approx & \left\{h_{\mathrm{r}}, W\right\}=\Delta h+\left(1-\frac{C}{2 A}-\frac{C}{2 B}\right) \Delta\left(\frac{J \sin g}{\sin I}\right) \\
& +e\left(1-\frac{C}{2 A}-\frac{C}{2 B}\right)\left\{\frac{J \sin (2 l+g)}{\sin I}, W_{\mathrm{K}}\right\} \\
\Delta I_{\mathrm{r}} \approx & \left\{I_{\mathrm{r}}, W\right\}=\Delta I+\left(1-\frac{C}{2 A}-\frac{C}{2 B}\right) \Delta(J \cos g) \\
& +e\left(1-\frac{C}{2 A}-\frac{C}{2 B}\right)\left\{J \cos (2 l+g), W_{\mathrm{K}}\right\} .
\end{aligned}
$$

Kinoshita (1977) neglected the bracket related to $W_{\mathrm{K}}$ and $e$ due to its smallness. However, we incorporate these terms in order to perform a full analysis of the indirect effect of the triaxiality. In addition, as it will be shown in the next section, it provides numerical contributions within the assumed truncation level. From Eqs. (12), (15) and (16) we get

$$
\begin{aligned}
&\left\{\frac{J}{\sin I} \sin (2 l+g), W_{\mathrm{K}}\right\}=-\frac{k}{\sin I} \sum_{\nu} \sum_{\varepsilon= \pm 1} C_{\nu}(\varepsilon) \\
& \times \frac{\sin \left(2 g+2 l-\varepsilon \Theta_{\nu}\right)}{n_{g}-\varepsilon N_{\nu}}, \\
&\left\{J \cos (2 l+g), W_{K}\right\}=-k \sum_{\nu} \sum_{\varepsilon= \pm 1} C_{\nu}(\varepsilon) \\
& \times \frac{\cos \left(2 g+2 l-\varepsilon \Theta_{\nu}\right)}{n_{g}-\varepsilon N_{\nu}} .
\end{aligned}
$$

Therefore, by considering Eqs. (21), (24), (26) and (27), the whole nutation, due to the indirect effect of the triaxiality of this plane turns out to be

$$
\begin{aligned}
\Delta_{\mathrm{e}} h_{\mathrm{r}}= & -\frac{e k}{\sin I}\left(1-\frac{C}{2 A}-\frac{C}{2 B}\right) \sum_{\nu} \sum_{\varepsilon= \pm 1} C_{\nu}(\varepsilon) \\
& \times \frac{\left(n_{g}+n_{l}-\varepsilon N_{\nu}\right) \sin \left(2 g+2 l-\varepsilon \Theta_{\nu}\right)}{\left(n_{g}-\varepsilon N_{\nu}\right)\left(n_{g}+2 n_{l}-\varepsilon N_{\nu}\right)}, \\
\Delta_{\mathrm{e}} I_{\mathrm{r}}= & -e k\left(1-\frac{C}{2 A}-\frac{C}{2 B}\right) \sum_{\nu} \sum_{\varepsilon= \pm 1} C_{\nu}(\varepsilon) \\
& \times \frac{\left(n_{g}+n_{l}-\varepsilon N_{\nu}\right) \cos \left(2 g+2 l-\varepsilon \Theta_{\nu}\right)}{\left(n_{g}-\varepsilon N_{\nu}\right)\left(n_{g}+2 n_{l}-\varepsilon N_{\nu}\right)} .
\end{aligned}
$$

Thus we remark that the nutation of the plane perpendicular to the rotational axis has also a quasi semi-diurnal period. The differential nutation of plane perpendicular to the rotational axis with respect to the nutation of the Andoyer plane is given by the so-called Oppolzer terms of the rotational axis (Kinoshita 1977). The indirect effect 
of the triaxiality on the nutation of the rotational axis is also contained in the corresponding Oppolzer terms, since in our order of approximation the Poisson terms are $\Delta_{\mathrm{e}} h=\Delta_{\mathrm{e}} I=0$. It is important to point out that, in this case, Oppolzer terms of the rotational axis are of the same order of magnitude as those of the figure axis, since the presence of the factor $1-C /(2 A)-C /(2 B)$ in Eq. (28) is compensated by the also small factor $n_{l}$ in Eq. (24) (see also Eq. (38) below). That behaviour is different from the usual one in Kinoshita's theory (1977) and subsequent works (Souchay et al. 1999), where Oppolzer terms of the rotational axis are smaller, by a factor of about $1 / 300$, than those of the figure axis. This is due to the fact that we consider the terms proportional to $e$ in Eq. (25). These terms are neglected by Kinoshita (1977) and in REN-2000 (Souchay et al. 1999).

\section{Numerical results}

In this section we compute numerically the additional nutations given by the formulae (24) and (28). In order to express these nutations in the same form as in Souchay et al. (1999), we must make some transformations. First, we note that the angle $g+l$ is reckoned from the $A$-principal axis of inertia of the Earth (Kinoshita 1977). However, the angle used in the nutation series, $\Phi$, is the angle of the sidereal rotation of the Earth (Bretagnon et al. 1997; Souchay et al. 1999). So, we must perform a phase shift given by the relationship (Souchay et al. 1999)

$\Phi=l+g+\Delta \Phi_{0}$

Here, $\Delta \Phi_{0}$ is the angle between Greenwich prime meridian and the $A$-principal axis, along the equatorial plane. To avoid misunderstandings, we determine $\Delta \Phi_{0}$ counterclockwise from the $A$-principal axis to the Greenwich meridian. Its numerical values can be derived from the $C_{22}$ and $S_{22}$ components of the geopotential (Souchay et al. 1999). Namely, we have

$\Delta \Phi_{0}=-\frac{1}{2} \arctan \frac{S_{22}}{C_{22}}=.2605521 \mathrm{rd}$

The minus sign appearing in the above formula is due to the fact that, with the values of $C_{22}$ and $S_{22}$ provided by the IERS standards (McCarthy 1992), the angle $1 / 2 \arctan \left(S_{22} / C_{22}\right)$ is reckoned from Greenwich to the $A$-principal axis, therefore, it has the opposite sign than that of the angle $\Delta \Phi_{0}$. This sign is not present in Souchay et al. (1999), or in Eq. (29), although the numerical results seem consistent with the arguments shown here. The present explanation also agrees with Eq. (12) from Bretagnon et al. (1997).
Taking into account the above considerations and putting $\varepsilon=-I, \varepsilon_{\mathrm{f}}=-I_{\mathrm{f}}, \varepsilon_{\mathrm{r}}=-I_{\mathrm{r}}, \psi_{\mathrm{f}}=-h_{\mathrm{f}}$ and $\psi_{\mathrm{r}}=-h_{\mathrm{r}}$, we have for the equatorial plane

$$
\begin{aligned}
\Delta_{\mathrm{e}} \psi_{\mathrm{f}}= & \frac{e k}{\sin \varepsilon} \sum_{\nu} \sum_{\varepsilon= \pm 1} \frac{n_{l} C_{\nu}(\varepsilon)}{\left(n_{g}-\varepsilon N_{\nu}\right)\left(n_{g}+2 n_{l}-\varepsilon N_{\nu}\right)} \\
& \times\left(\cos \left(2 \Delta \Phi_{0}\right) \sin \left(2 \Phi-\varepsilon \Theta_{\nu}\right)\right. \\
& \left.-\sin \left(2 \Delta \Phi_{0}\right) \cos \left(2 \Phi-\varepsilon \Theta_{\nu}\right)\right) \\
\Delta_{\mathrm{e}} \varepsilon_{\mathrm{f}}= & -e k \sum_{\nu} \sum_{\varepsilon= \pm 1} \frac{n_{l} C_{\nu}(\varepsilon)}{\left(n_{g}-\varepsilon N_{\nu}\right)\left(n_{g}+2 n_{l}-\varepsilon N_{\nu}\right)} \\
& \times\left(\cos \left(2 \Delta \Phi_{0}\right) \cos \left(2 \Phi-\varepsilon \Theta_{\nu}\right)\right. \\
& \left.+\sin \left(2 \Delta \Phi_{0}\right) \sin \left(2 \Phi-\varepsilon \Theta_{\nu}\right)\right)
\end{aligned}
$$

and for the plane perpendicular to the rotational axis

$$
\begin{aligned}
\Delta_{\mathrm{e}} \psi_{\mathrm{r}}= & -\frac{e k}{\sin \varepsilon}\left(1-\frac{C}{2 A}-\frac{C}{2 B}\right) \sum_{\nu} \sum_{\varepsilon= \pm 1} \frac{C_{\nu}(\varepsilon)}{\left(n_{g}-\varepsilon N_{\nu}\right)} \\
& \times \frac{\left(n_{g}+n_{l}-\varepsilon N_{\nu}\right)}{\left(n_{g}+2 n_{l}-\varepsilon N_{\nu}\right)}\left(\cos \left(2 \Delta \Phi_{0}\right) \sin \left(2 \Phi-\varepsilon \Theta_{\nu}\right)\right. \\
& \left.-\sin \left(2 \Delta \Phi_{0}\right) \cos \left(2 \Phi-\varepsilon \Theta_{\nu}\right)\right) \\
\Delta_{\mathrm{e}} \varepsilon_{\mathrm{r}}= & e k\left(1-\frac{C}{2 A}-\frac{C}{2 B}\right) \sum_{\nu} \sum_{\varepsilon= \pm 1} \frac{C_{\nu}(\varepsilon)}{\left(n_{g}-\varepsilon N_{\nu}\right)} \\
& \times \frac{\left(n_{g}+n_{l}-\varepsilon N_{\nu}\right)}{\left(n_{g}+2 n_{l}-\varepsilon N_{\nu}\right)}\left(\cos \left(2 \Delta \Phi_{0}\right) \cos \left(2 \Phi-\varepsilon \Theta_{\nu}\right)\right. \\
& \left.+\sin \left(2 \Delta \Phi_{0}\right) \sin \left(2 \Phi-\varepsilon \Theta_{\nu}\right)\right)
\end{aligned}
$$

To evaluate these series we need the numerical values of $k$ for the Moon and the Sun, respectively $k_{\mathrm{M}}$ and $k_{\mathrm{S}}$, that will be taken from Souchay \& Kinoshita (1996). We need also the coefficients $C_{\nu}(\varepsilon), N_{\nu}$ that will be obtained from Kinoshita (1977) and Kinoshita \& Souchay (1990), the angular velocity of the Earth, $\omega_{\mathrm{E}} \simeq \dot{\Phi}$, that will be taken from Aoki et al. (1982) and the obliquity $\varepsilon$, at epoch J2000, taken from Lieske et al. (1977). With respect to the remaining parameters let us point out that in order to derive their numerical values we will introduce another parameter related to the triaxiality of the Earth

$\eta=\frac{B-A}{2 C-A-B}$

which is more convenient than $e$. Then, we can express the moments of inertia in terms of $\eta$ and the dynamical ellipticity $H_{\mathrm{d}}$ (see Eq. (1)). So, we have

$\frac{C}{A}=\frac{1}{1-H_{\mathrm{d}}(\eta+1)}, \frac{B}{A}=\frac{1+H_{\mathrm{d}}(\eta-1)}{1-H_{\mathrm{d}}(\eta+1)}$. 
Table 1. Numerical parameters used in this work.

\begin{tabular}{lcl}
\hline \hline Parameter & Value & Source \\
\hline$\varepsilon$ & $0.4090928041 \mathrm{rd}$ & Lieske et al. (1977) \\
$\dot{\Phi}$ & $230121.67526278 \mathrm{rd} / \mathrm{cy}$ & Aoki et al. (1982) \\
$J_{2}$ & $-1082.626075 \times 10^{-6}$ & IERS Standards (1992) \\
$C_{22}$ & $1.574410 \times 10^{-6}$ & IERS Standards (1992) \\
$S_{22}$ & $-.903757 \times 10^{-6}$ & IERS Standards (1992) \\
$k_{\mathrm{M}}$ & $7546^{\prime \prime} .717329 / \mathrm{cy}$ & Souchay et al. (1996) \\
$k_{\mathrm{S}}$ & $3475^{\prime \prime} 413512 / \mathrm{cy}$ & Souchay et al. (1996) \\
$H_{\mathrm{d}}$ & .0032737548 & Souchay et al. (1996) \\
\hline
\end{tabular}

From these relationships, (1) and (4), we can obtain the values of $e, n_{l}$ and $n_{g}$. In addition, it will also be useful to put

$n_{l}=\omega_{\mathrm{E}}-n_{g},\left(1-\frac{C}{2 A}-\frac{C}{2 B}\right)=\frac{n_{l}}{\omega_{\mathrm{E}}}=1-\frac{n_{g}}{\omega_{\mathrm{E}}}$,

where (Kinoshita 1977) $\tilde{G} \simeq \tilde{L} \simeq C \omega_{\mathrm{E}}$.

The dynamical ellipticity $H_{\mathrm{d}}$ will be also taken from Souchay \& Kinoshita (1996). $\eta$ is computed from the coefficients of the geopotential (Bretagnon et al. 1997):

$\frac{2 C-A-B}{2 m_{\mathrm{E}} a_{\mathrm{E}}^{2}}=J_{2}, \frac{B-A}{m_{\mathrm{E}} a_{\mathrm{E}}^{2}}=4 \sqrt{C_{22}^{2}+S_{22}^{2}}$,

where $J_{2}$ is the zonal harmonic of the second order. With these definitions and by means of the values in Table 1 we get

$$
\begin{aligned}
& \eta=.0033536292, e=.0033646441, \\
& \frac{n_{g}}{\omega_{\mathrm{E}}}=1.0032845076 .
\end{aligned}
$$

The parameters shown in Table 1 are the same as those used by Souchay et al. (1999). With these parameters and those of Eq. (37) the amplitudes of the nutation series due to the indirect effect of the triaxiality are computed. Since the indirect effect of the triaxiality is small, its contribution will be within the truncation level only for the leading arguments $\Theta_{\nu}$, that is to say, those corresponding to larger amplitudes. For convenience, we have performed the calculations using the whole set of 107 arguments $\Theta_{\nu}$ given by Kinoshita (1977), which obviously contain all the leading terms relevant for this study. In Table 2 we show the series in longitude corresponding to the plane of figure and the plane normal to the rotational axis. In Table 3 we show the series in obliquity corresponding to these same planes. We have displayed all the terms larger than $0.005 \mu$ as.

As one could expect, the amplitudes of the nutations terms due to the indirect effect of the triaxiality of the equatorial plane and the plane perpendicular to the rotational axis are small. Besides, from these values it is clear that the indirect effect of the triaxiality on other geopotential coefficients different from $J_{2}$ can be neglected. We have found eight terms larger than $0.05 \mu$ as (five terms in longitude and three in obliquity for nutations of the equatorial plane). It is also worthy noting that the argument of the nutation is the same as the argument that appears when treating the influence of the $C_{22}$ and $S_{22}$ coefficients on the nutations (Souchay et al. 1999). So, these new contributions should be added to those of REN-2000 tables (Souchay et al. 1999).

To make easier the visualization of the amount of the indirect effect, we have gathered in Table 4 the largest terms for each different contribution to the nutation of the figure axis of the rigid Earth having a quasi semidiurnal period, including the usual "direct effects" as well as the new "indirect effect" studied in this work. The direct contributions arise from the non-zonal harmonics of second order and second, third and fourth degrees and are taken from REN-2000 (Souchay et al. 1999). For each nutation series of the figure axis coming from a selected harmonic, we have chosen the largest term in longitude and in obliquity. Explicitly, the largest term due to the harmonics $C_{22}$ and $S_{22}$ is taken from Souchay et al. (1999), the one given by the harmonics $C_{32}$ and $S_{32}$ is computed from Folgueira et al. (1998a) and the one due to the harmonics $C_{42}$ and $S_{42}$ is taken from Folgueira et al. (1998b). In the last row we have also displayed the largest nutation term of the figure axis caused by the indirect effect of the triaxiality $\left(J_{2}\right.$ indirect effect). As can be seen, the largest contribution of the indirect effect is smaller than that of the harmonics $C_{32}$ and $S_{32}$ and much smaller than that of the harmonics $C_{22}$ and $S_{22}$, which is the leading one. However, the indirect effect contribution is much more important than the direct effect due to the harmonics $C_{42}$ and $S_{42}$.

For the nutations of the plane perpendicular to the rotational axis there are five terms in longitude and three in obliquity that are larger than $0.05 \mu$ as. Thus, we also have quasi semi-diurnal nutations for the rotational axis within the sub-microarcsecond level. The amplitude of these terms is mainly caused by the part that arises from $W_{\mathrm{K}}$, Eq. (27).

It is also interesting to point out that the nutations of the figure axis and of the rotational axis due to the indirect effect of the triaxiality have almost the same values but opposite sign. The explanation of this fact can be derived by considering the analytical expressions of these terms. The ratio between the nutation in longitude (in sine or cosine) for the figure axis, Eq. (31), and for the rotational

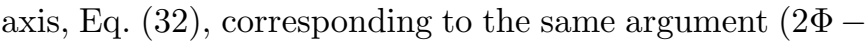
$\left.\varepsilon \Theta_{\nu}\right)$ can be written as

$$
\begin{aligned}
\left.\frac{\Delta_{\mathrm{e}} \psi_{\mathrm{r}}}{\Delta_{\mathrm{e}} \psi_{\mathrm{f}}}\right|_{\left(2 \Phi-\varepsilon \Theta_{\nu}\right)}= & -\left(1-\frac{C}{2 A}-\frac{C}{2 B}\right) \\
& \times \frac{\left(n_{g}+n_{l}-\varepsilon N_{\nu}\right)}{n_{l}} \\
= & -\frac{n_{l}\left(\omega_{\mathrm{E}}-\varepsilon N_{\nu}\right)}{n_{l} \omega_{\mathrm{E}}}=-1+\varepsilon \frac{N_{\nu}}{\omega_{\mathrm{E}}}
\end{aligned}
$$

where we have taken into account Eq. (35). This ratio is very close to -1 for most of the terms. The same conclusion is obtained for the nutation in obliquity. 
Table 2. Indirect effect of the triaxiality. Figure and rotational axes. Longitude (Unit $=\mu$ as).

\begin{tabular}{rcccccccccrr}
\hline \hline & \multicolumn{4}{c}{ Argument } & \multicolumn{2}{c}{ Period } & \multicolumn{2}{c}{ Figure axis } & \multicolumn{2}{c}{ Rotational axis } & Alias \\
\hline$\Phi$ & $l_{\mathrm{M}}$ & $l_{\mathrm{S}}$ & $F$ & $D$ & $\Omega$ & $($ days $)$ & $\Delta_{e} \psi_{f}(\sin )$ & $\Delta_{\mathrm{e}} \psi_{\mathrm{f}}(\cos )$ & $\Delta_{\mathrm{e}} \psi_{\mathrm{r}}(\sin )$ & $\Delta_{\mathrm{e}} \psi_{\mathrm{r}}(\cos )$ & period \\
\hline 2 & 0 & 0 & 0 & 0 & 0 & .498635 & -.210 & .120 & .210 & -.120 & $\infty$ \\
2 & 0 & 0 & 0 & 0 & -1 & .498598 & -.028 & .016 & .028 & -.016 & -6798.36 \\
2 & 0 & 0 & -2 & 2 & -2 & .500000 & .070 & -.040 & -.070 & .040 & 182.62 \\
2 & -1 & 0 & 0 & 0 & 0 & .507826 & -.013 & .007 & .012 & -.007 & 27.55 \\
2 & 0 & 0 & -2 & 0 & -2 & .517526 & .173 & -.099 & -.161 & .092 & 13.66 \\
2 & 0 & 0 & -2 & 0 & -1 & .517569 & .033 & -.019 & -.030 & .017 & 13.63 \\
2 & -1 & 0 & -2 & 0 & -2 & .527441 & .036 & -.021 & -.032 & .018 & 9.13 \\
2 & 1 & 0 & 0 & 0 & 0 & .489770 & -.011 & .006 & .011 & -.006 & -27.55 \\
2 & 0 & 0 & 2 & 0 & 2 & .481037 & -.006 & .003 & .006 & -.003 & -13.66 \\
\hline
\end{tabular}

Table 3. Indirect effect of the triaxiality. Figure and rotational axes. Obliquity (Unit $=\mu$ as).

\begin{tabular}{rcccccccccrr}
\hline \hline & \multicolumn{4}{c}{ Argument } & & Period & \multicolumn{2}{c}{ Figure axis } & \multicolumn{2}{c}{ Rotational axis } & Alias \\
\hline$\Phi$ & $l_{\mathrm{M}}$ & $l_{\mathrm{S}}$ & $F$ & $D$ & $\Omega$ & $($ days $)$ & $\Delta_{\mathrm{e}} \varepsilon_{\mathrm{f}}(\sin )$ & $\Delta_{\mathrm{e}} \varepsilon_{\mathrm{f}}(\cos )$ & $\Delta_{\mathrm{e}} \varepsilon_{\mathrm{r}}(\sin )$ & $\Delta_{\mathrm{e}} \varepsilon_{\mathrm{r}}(\cos )$ & period \\
\hline 2 & 0 & 0 & 0 & 0 & 0 & .498635 & .048 & .083 & -.048 & -.083 & $\infty$ \\
2 & 0 & 0 & 0 & 0 & -1 & .498598 & .006 & .011 & -.006 & -.011 & -6798.36 \\
2 & 0 & 0 & -2 & 2 & -2 & .500000 & -.016 & -.028 & .016 & .028 & 182.62 \\
2 & 0 & 0 & -2 & 0 & -2 & .517526 & -.040 & -.069 & .037 & .064 & 13.66 \\
2 & 0 & 0 & -2 & 0 & -1 & .517569 & -.007 & -.013 & .007 & .012 & 13.63 \\
2 & -1 & 0 & -2 & 0 & -2 & .527441 & -.008 & -.014 & .007 & .013 & 9.13 \\
\hline
\end{tabular}

Table 4. Comparison of the largest quasi semi-diurnal amplitudes for direct and indirect effects. Figure axis (Unit $=\mu$ as).

\begin{tabular}{cccccccccccccc}
\hline \hline & \multicolumn{1}{c}{ Argument } & \multicolumn{1}{c}{ Period } & \multicolumn{2}{c}{ Longitude } & \multicolumn{2}{c}{ Obliquity } & Harmonic & Type & Source \\
\hline \multicolumn{1}{c}{$\Delta \psi_{\mathrm{f}}$} & \multicolumn{1}{c}{$\Delta \varepsilon_{\mathrm{f}}$} \\
\hline$\Phi$ & $l_{\mathrm{M}}$ & $l_{\mathrm{S}}$ & $F$ & $D$ & $\Omega$ & (days) & $\sin$ & $\cos$ & $\sin$ & $\cos$ \\
\hline 2 & 0 & 0 & 0 & 0 & 0 & .49864 & 31.3 & -18.0 & -7.1 & -12.4 & $C_{22}, S_{22}$ & direct & REN-2000 \\
2 & 0 & 0 & -1 & 0 & -1 & .50790 & -.244 & -.356 & -.097 & .067 & $C_{32}, S_{32}$ & direct & REN-2000 \\
2 & 0 & 0 & 1 & 0 & 2 & .48973 & -.008 & -.012 & -.114 & .078 & $C_{32}, S_{32}$ & direct & REN-2000 \\
2 & 0 & 0 & 0 & 0 & 0 & .49864 & .016 & .030 & - & - & $C_{42}, S_{42}$ & direct & REN-2000 \\
2 & 0 & 0 & 0 & 0 & 0 & .49864 & -.210 & .120 & .048 & .083 & $J_{2}$ & indirect & This work \\
\hline
\end{tabular}

Finally, let us point out that the internal structure of the Earth could amplify these effects due to the resonance caused by the fluid core, that could reach even a few microarcseconds. So, this discussion should be extended to non-rigid Earth models, in the same way as Getino et al. (2001), who studied the effect of the $C_{22}$ and $S_{22}$ coefficients on the nutations of a two layer Earth model. This work is in progress and will be presented in a forthcoming paper.

Acknowledgements. This work has been partially supported by Spanish Projects Ministerio de Ciencia y Tecnología, $I+D$, Project No. AYA2000-1787, Project No. AYA20010787, PNE-015/2001-C and Junta de Castilla y León Project VA72/02.

\section{References}

Aoki, S., Guinot, B., Kaplan, G., et al. 1982, A\&A, 105, 359 Bretagnon, P., Rocher, P., \& Simon, J. L. 1997, A\&A, 319, 305
Folgueira, M., Souchay, J., \& Kinoshita, H. 1998a, Celes. Mech., 69, 373

Folgueira, M., Souchay, J., \& Kinoshita, H. 1998b, Celes. Mech., 70, 147

Getino, J., Ferrándiz, J. M., \& Escapa, A. 2001, A\&A, 370, 330

Goldstein, H. 1980, Classical Mechanics, 2nd ed. (AddisonWesley, Reading, MA)

Hori, G. 1966, PASJ, 18, 287

Kinoshita, H. 1972, PASJ, 24, 423

Kinoshita, H. 1977, Celes. Mech., 15, 277

Kinoshita, H., \& Souchay, J. 1990, Celes. Mech., 48, 187

Lieske, J. H., Lederle, T., Fricke, W., \& Morando, B. 1977, A\&A, 58, 1

McCarthy, D. D. 1992, IERS Standards

Souchay, J. 2000, Proc. IAU Coll., 180, 190

Souchay, J., \& Kinoshita, H. 1996, A\&A, 312, 1017

Souchay, J., \& Kinoshita, H. 1997, A\&A, 318, 639

Souchay, J., Losley, B., Kinoshita, H., \& Folgueira, M. 1999, ApJS, 135, 111 